\title{
Continuous excitations of the triangular-lattice quantum spin liquid $\mathrm{YbMgGaO}_{4}$
}

\author{
Joseph A. M. Paddison ${ }^{1}$, Marcus Daum ${ }^{1 \dagger}$, Zhiling Dun ${ }^{2 \dagger}$, Georg Ehlers ${ }^{3}$, Yaohua Liu ${ }^{3}$, \\ Matthew B. Stone ${ }^{3}$, Haidong Zhou $^{2}$ and Martin Mourigal ${ }^{1 \star}$
}

\begin{abstract}
A quantum spin liquid (QSL) is an exotic state of matter in which electrons' spins are quantum entangled over long distances, but do not show magnetic order in the zero-temperature limit'. The observation of QSL states is a central aim of experimental physics, because they host collective excitations that transcend our knowledge of quantum matter; however, examples in real materials are scarce $^{2}$. Here, we report neutron-scattering experiments on $\mathrm{YbMgGaO}_{4}$, a QSL candidate in which $\mathrm{Yb}^{3+}$ ions with effective spin-1/2 occupy a triangular lattice ${ }^{3-6}$. Our measurements reveal a continuum of magnetic excitationsthe essential experimental hallmark of a QSL7 - at very low temperature $(0.06 \mathrm{~K})$. The origin of this peculiar excitation spectrum is a crucial question, because isotropic nearestneighbour interactions do not yield a QSL ground state on the triangular lattice ${ }^{8}$. Using measurements in the field-polarized state, we identify antiferromagnetic next-nearest-neighbour interactions $^{9-12}$, spin-space anisotropies ${ }^{4,10,13,14}$, and chemical disorder ${ }^{15}$ between the magnetic layers as key ingredients in $\mathrm{YbMgGaO}_{4}$.
\end{abstract}

The phenomenon of entanglement is one of the fundamental results of quantum mechanics. Among its most extraordinary manifestations are quantum spin liquids, in which a macroscopic number of spins are entangled but do not show conventional magnetic order ${ }^{1}$. The earliest examples of QSLs were found in quasi-onedimensional systems such as Heisenberg spin chains ${ }^{16}$ and in the distorted triangular-lattice antiferromagnet $\mathrm{Cs}_{2} \mathrm{CuCl}_{4}$ (ref. 17). The search for QSLs in two- and three-dimensional systems has focused on frustrated magnets, in which the lattice geometry prevents all magnetic interactions from being satisfied simultaneously ${ }^{1,2}$ or in which spin-orbit coupling leads to interactions that depend on bond directions ${ }^{18}$. In two dimensions, the prototype of a QSL is Anderson's 'resonating valence bond' model ${ }^{19}$. Its ground state is a superposition of all possible tilings of dimers on the triangular lattice, where each dimer is built from an entangled pair of spin$1 / 2$; its excitations are unpaired spin- $1 / 2$, which are delocalized. The presence of delocalized excitations with fractional quantum numbers leads to a necessary experimental signature of a QSL: a magnetic excitation spectrum that is continuous in energy but structured in momentum space ${ }^{7}$. Such a spectrum has indeed been observed using neutron-scattering measurements of $\mathrm{ZnCu}_{3}(\mathrm{OD})_{6} \mathrm{Cl}_{2}$ ('herbertsmithite' $)^{7,20}$, in which spins occupy a kagome lattice. However, the stabilization of a continuous excitation spectrum by quantum fluctuations in a structurally perfect triangular-lattice magnetthe scenario originally proposed by Anderson ${ }^{19}$ - has remained an open question.
Recent experiments have identified the triangular-lattice magnet $\mathrm{YbMgGaO}_{4}$ as an exciting QSL candidate ${ }^{3,4}$. The crystal structure (space group $R \overline{3} \mathrm{~m}$ ) contains undistorted triangular planes of magnetic $\mathrm{Yb}^{3+}$ (Fig. 1a), separated by two triangular planes occupied in a disordered manner by $\mathrm{Mg}^{2+}$ and $\mathrm{Ga}^{3+}$ (Fig. 1b) . Thermodynamic and muon-spin relaxation $(\mu \mathrm{SR})^{5}$ measurements show the absence of conventional magnetic order and spin freezing to $T<0.1 \mathrm{~K}$, far below the Weiss temperature $\theta_{\mathrm{W}} \approx-4 \mathrm{~K}$; moreover, the apparent absence of zero-point entropy indicates that the system essentially occupies a single quantum state at $0.06 \mathrm{~K}$ (ref. 3). The crystal-field ground state of $\mathrm{Yb}^{3+}$ is a Kramers doublet ${ }^{4}$, separated by a large energy gap of $38 \mathrm{meV}$ from the first excited state (Fig. 1c and Supplementary Fig. 1); hence, an effective spin- $1 / 2$ description is appropriate at low temperature, as for 'quantum spin ice' $\mathrm{Yb}_{2} \mathrm{Ti}_{2} \mathrm{O}_{7}$ (ref. 21). Electron-spin resonance (ESR) measurements indicate that the nearest-neighbour magnetic interaction is anisotropic and depends on the bond orientation ${ }^{4}$. This provides one potential mechanism to stabilize a QSL ground state on the triangular lattice ${ }^{13,14}$. However, several alternative mechanisms for QSL behaviour are known, including furtherneighbour interactions ${ }^{9-12}$, disorder-induced entanglement ${ }^{15}$ and multi-spin interactions ${ }^{22}$.

Experiments to reveal the nature of the potential triangularlattice QSL in $\mathrm{YbMgGaO}_{4}$ are therefore crucial. To enable such experiments, we have grown a large single crystal of $\mathrm{YbMgGaO}_{4}$ using the floating-zone technique (Supplementary Fig. 2). We characterized our crystal using specific heat, magnetization, and single-crystal neutron-diffraction measurements. In addition, we compared specific-heat and X-ray diffraction measurements of crushed crystal pieces with the same measurements of a powder sample prepared by the solid-state method of ref. 3. Single-crystal neutron-diffraction measurements (Supplementary Fig. 3) reveal that our single crystal is predominantly a single grain and is therefore suitable for inelastic neutron-scattering measurements. The magnetic specific heat shows a broad peak at $T \approx 3 \mathrm{~K}$ in zero magnetic field, and a $T^{\gamma}$ dependence with $\gamma=0.70(1)$ below the peak (Fig. 1d), consistent with previous experimental results $^{3}$. Our magnetic entropy (Fig. 1e) and magnetization (Fig. 1f) measurements fully support the effective spin-1/2 scenario (with $\left.g_{\|} \approx 3.7\right)$ for $\mathrm{Yb}^{3+}$ at low temperatures. Rietveld refinements to our X-ray diffraction data (Supplementary Fig. 4) yield a good fit for the published structural model ${ }^{3}$ with no $\mathrm{Yb}^{3+} / \mathrm{Ga}^{3+}$ site mixing observed within our experimental accuracy and with disordered occupancy on the interstitial layers by $\mathrm{Mg}^{2+}$ and $\mathrm{Ga}^{3+}$ (Supplementary Table 1). Such disorder can affect

\footnotetext{
${ }^{1}$ School of Physics, Georgia Institute of Technology, Atlanta, Georgia 30332, USA. ${ }^{2}$ Department of Physics and Astronomy, University of Tennessee, Knoxville, Tennessee 37996, USA. ${ }^{3}$ Quantum Condensed Matter Division, Oak Ridge National Laboratory, Oak Ridge, Tennessee 37831 , USA. ${ }^{\dagger}$ These authors contributed equally to this work. *e-mail: mourigal@gatech.edu
} 

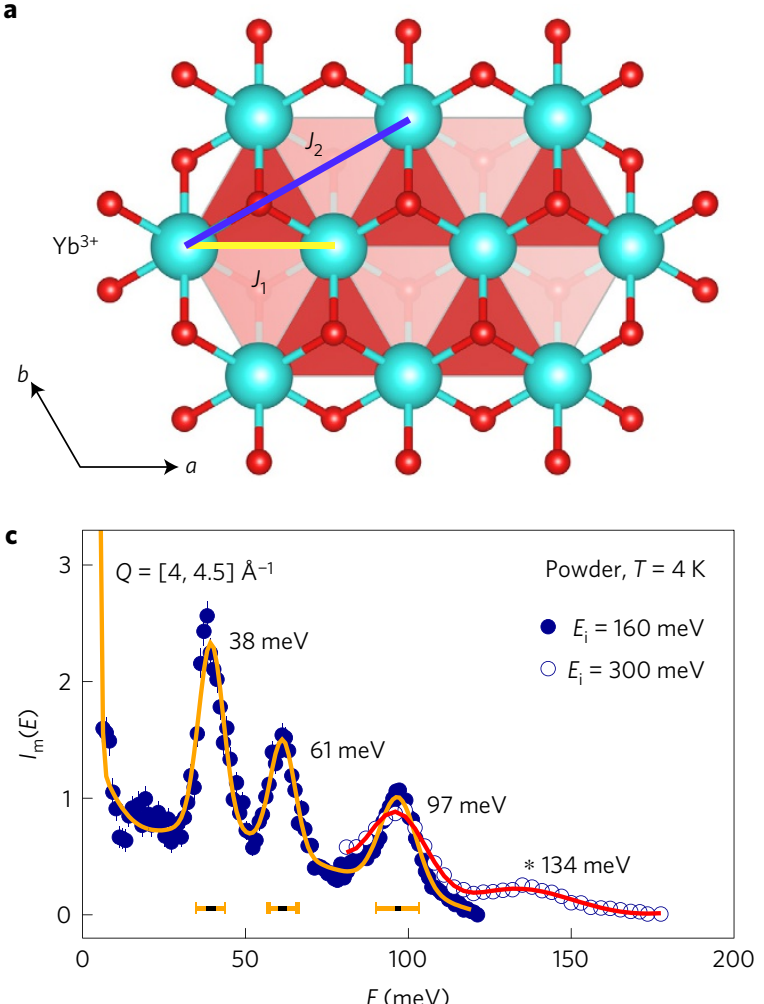

d

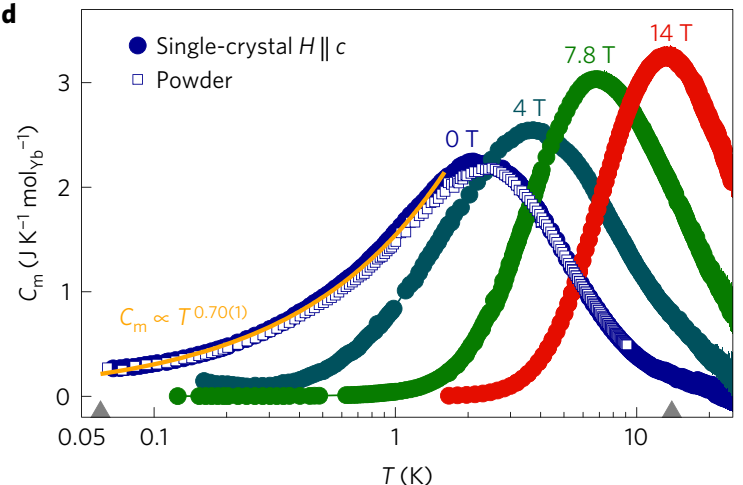

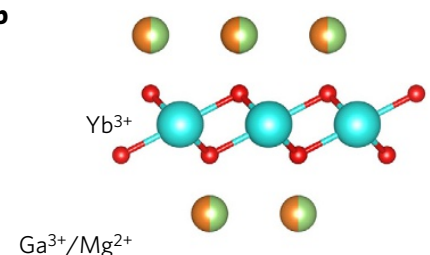

$\mathrm{Ga}^{3+} / \mathrm{Mg}^{2+}$
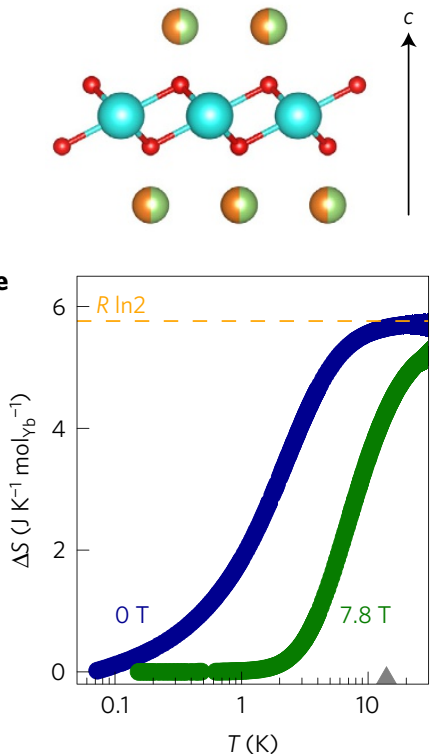

f

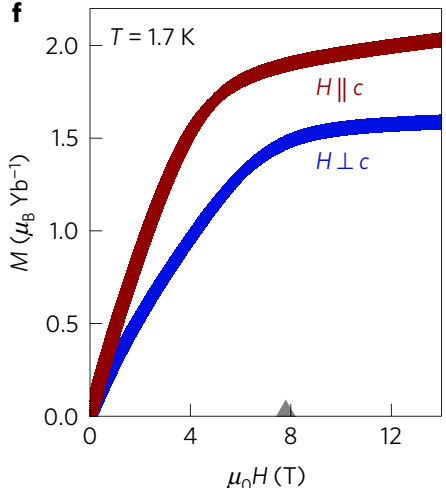

Figure 1 | Structure and magnetic properties of $\mathrm{YbMgGaO}_{4}$. a, Partial crystal structure, showing a triangular layer of $\mathrm{Yb}^{3+}$ ions (large cyan spheres) and their coordination by oxygen (small red spheres). A nearest-neighbour interaction pathway $J_{1}$ and a next-nearest-neighbour interaction pathway $J_{2}$ are shown by yellow and blue lines, respectively. $\mathbf{b}$, Stacking of the $\mathrm{Yb}^{3+}$ planes along the $\mathrm{c}$ axis and their separation by two layers of disordered $\mathrm{Mg}^{2+}$ and $\mathrm{Ga}^{3+}$ ions. c, High-energy neutron-scattering measurements on a powder sample at $T=4 \mathrm{~K}$. Filled (respectively, open) symbols correspond to an incident neutron energy of $160 \mathrm{meV}$ (respectively, $300 \mathrm{meV}$ ). Solid lines are Gaussian fits to the observed magnetic excitations. Light (respectively, dark) horizontal bars correspond to the observed (respectively, calculated) peak's full-width at half-maximum. $\mathbf{d}$, Magnetic component of the specific heat $C_{m}(T)$, showing data measured on a powder sample (open squares) and a single-crystal sample (filled circles). Single-crystal data are shown for applied magnetic fields along the $c$ axis of $0,4,7.8$ and $14 \mathrm{~T}$ (labelled above each curve). The orange line shows a fit of the zero-field single-crystal data to a power law, $C_{m}(T) \propto T^{0.70(1)}$.e, Magnetic entropy change $\Delta S_{m}$, showing data measured in zero field and in a $7.8 T$ field (labelled on each curve). $\mathbf{f}$, Dependence of the magnetization $\mathrm{M}$ on applied field $\mu_{0} \mathrm{H}$, showing data measured at temperatures of $1.7 \mathrm{~K}$ for two different field directions (labelled on each curve). In $\mathbf{d}$-f the temperatures and applied fields at which we performed neutron-scattering measurements (Figs 2 and 3 ) are indicated by grey arrowheads. Error bars correspond to 1 standard error.

magnetism by modifying the charge environment around $\mathrm{Yb}^{3+}$ ions. Our broadband neutron-scattering experiment (Fig. 1c and Supplementary Fig. 1) reveals three intense crystal electric-field excitations at 38(1), 61(1) and 97(1) $\mathrm{meV}$, consistent with the four Kramers doublets expected for $\mathrm{Yb}^{3+}$. These excitations are broader than the instrumental resolution and an additional weak mode is observed around $134(8) \mathrm{meV}$, hinting at a distribution of local environments across $\mathrm{Yb}^{3+}$ ions. In all our measurements, we observe no significant differences between powder and single-crystal samples, suggesting that the sample dependence that has afflicted
QSL candidates such as $\mathrm{Yb}_{2} \mathrm{Ti}_{2} \mathrm{O}_{7}$ (ref. 23) is not evident in $\mathrm{YbMgGaO}_{4}$.

Neutron-scattering experiments measure spin correlations as a function of energy and reciprocal space, yielding direct information about correlated quantum states. Single-crystal neutronscattering data measured in zero field are shown in Fig. 2, and provide strong evidence for QSL behaviour. Throughout, we plot neutron-scattering intensity as $\left(1+\mathrm{e}^{-\beta E}\right) I(\mathbf{Q}, E)$, where $I(\mathbf{Q}, E)$ is the measured intensity as a function of scattering wavevector $\mathbf{Q}=h \boldsymbol{a}^{*}+k \boldsymbol{b}^{*}+l \boldsymbol{c}^{*}$ and energy transfer $E$, and the factor $1+\mathrm{e}^{-\beta E}$ 


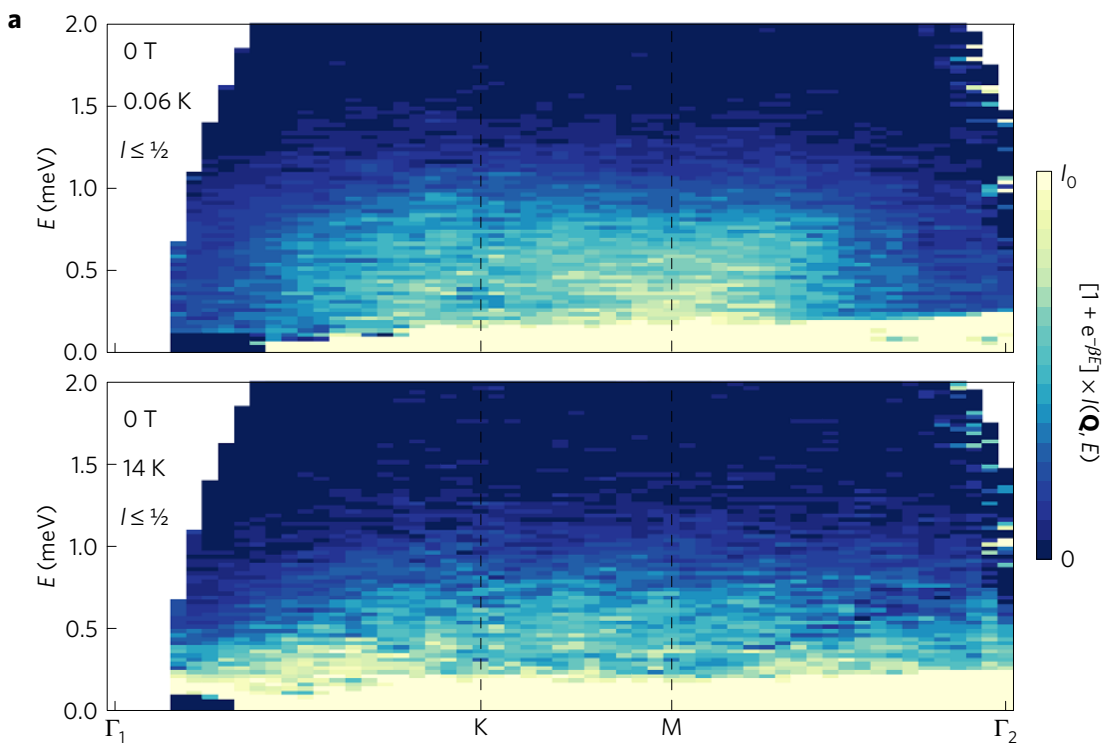

b

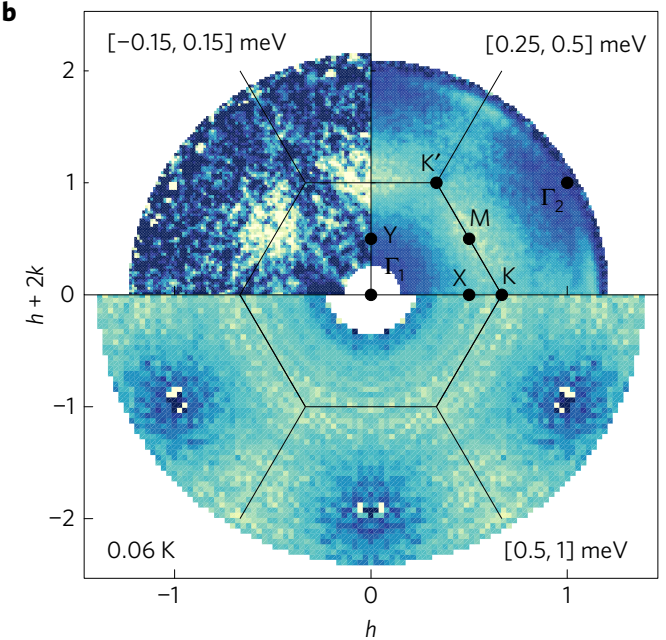

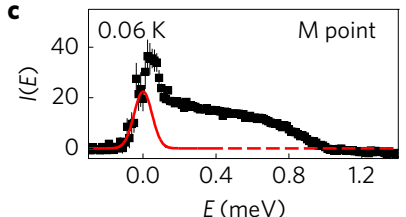

d

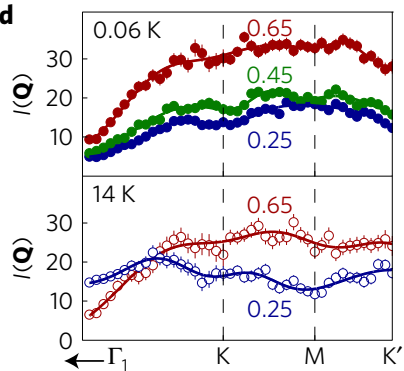

Figure 2 | Neutron-scattering data for $\mathrm{YbMgGaO}_{4}$ measured in zero applied field. a, Energy dependence of magnetic excitations along high-symmetry directions in reciprocal space, showing data at $0.06 \mathrm{~K}$ (upper panel) and $14 \mathrm{~K}$ (lower panel). Reciprocal-space points are labelled in $\mathbf{b}$ and scattering intensity in arbitrary units is shown as a colour scale. The temperature of $0.06 \mathrm{~K}$ was measured at the mixing chamber of our dilution refrigerator. b. Wavevector dependence of magnetic excitations at $0.06 \mathrm{~K}$ integrated for different energy transfers: scattering within the elastic instrumental resolution (upper left panel), 0.25 to $0.5 \mathrm{meV}$ (upper right panel) and 0.5 to $1.0 \mathrm{meV}$ (lower panel). c, Energy dependence of the scattering at the $\mathrm{M}$ point. The red line corresponds to the amount of scattering within the $0.11 \mathrm{meV}$ elastic resolution of the instrument. d, One-dimensional cuts along high-symmetry directions at $0.06 \mathrm{~K}$ (upper panel) and $14 \mathrm{~K}$ (lower panel). Each panel shows energy transfers of $0.25,0.45$ and 0.65 meV (labelled on the graph). For a,b upper panels, $\mathbf{c}$ and $\mathbf{d}$, the incident neutron energy $E_{\mathrm{i}}=3.32 \mathrm{meV}$ and the data have been integrated over the vertical range $-\frac{1}{2} \leq 1 \leq \frac{1}{2}$; for $\mathbf{b}$ lower panel, $E_{i}=12 \mathrm{meV}$ and $-1 \leq I \leq 1$. For $\mathbf{b}-\mathbf{d}$, the $7.8 \mathrm{~T}$ data (see below) are used as background. The scattering intensity in the top panels of $\mathbf{b}$ is multiplied by a factor $2 / 3$ to share the same colour scale as $\mathbf{a}$. Error bars correspond to 1 standard error.

corrects for detailed balance ${ }^{24}$. Figure 2 a shows the energy dependence of the scattering intensity along high-symmetry reciprocalspace directions. The scattering at $E \gtrsim 0.2 \mathrm{meV}$ is magnetic, as shown by its dependence on applied magnetic field (discussed below). At both $0.06 \mathrm{~K}$ and $14 \mathrm{~K}$, our data reveal a broad continuum of excitations, in contrast to the spin-wave scattering observed in the spin-1/2 triangular-lattice compound $\mathrm{Ba}_{3} \mathrm{CoSb}_{2} \mathrm{O}_{9}$ (ref. 25). The excitation continuum has a bandwidth of $1.3 \mathrm{meV}$ and is gapless within the experimental energy resolution of approximately $0.1 \mathrm{meV}$. This scattering spans a much smaller energy scale but is otherwise qualitatively similar to herbertsmithite 7,20 - a surprising result, because the kagome lattice of herbertsmithite is considered more highly frustrated than the triangular lattice of $\mathrm{YbMgGaO}_{4}$ (refs 8,26).

The wavevector dependence of the $0.06 \mathrm{~K}$ scattering intensity is shown in Fig. 2b (see also Supplementary Fig. 5). The elastic scattering is much broader than the instrumental resolution $(\delta Q / Q \approx 2 \%)$ and reveals no sharp magnetic Bragg peaks, confirming the absence of long-range magnetic order at $0.06 \mathrm{~K}$. The low-energy scattering $(E \leq 0.5 \mathrm{meV})$ shows a broad maximum at the $\mathrm{M} \equiv\left(\frac{1}{2} 00\right)$ point of the hexagonal Brillouin zone, whereas at higher energies $(0.5 \leq E \leq 1.0 \mathrm{meV})$ the scattering is isotropic around the zone boundary. The energy dependence of the magnetic signal at the $\mathrm{M}$ point (Fig. 2c) indicates that at most $16(3) \%$ of the total spectral weight is elastic. Correspondingly, the inelastic contribution $(\geq 84(3) \%)$ is large compared with the $66 \%$ expected for a spin$1 / 2$ glass $^{27}$. This rules out a totally frozen state for $\mathrm{YbMgGaO}_{4}$, in agreement with $\mu \mathrm{SR}^{5}$. Further insight into the M-point scattering maximum is obtained from line plots at different energies (Fig. 2d). At $0.06 \mathrm{~K}$, these plots reveal a weak dispersive excitation originating from the M point, superimposed on the continuum shown in Fig. 2a. Strikingly, some structure persists in the excitation spectrum at $14 \mathrm{~K}\left(\gtrsim 3 \theta_{\mathrm{W}}\right)$, but the location of the intensity maximum at low 

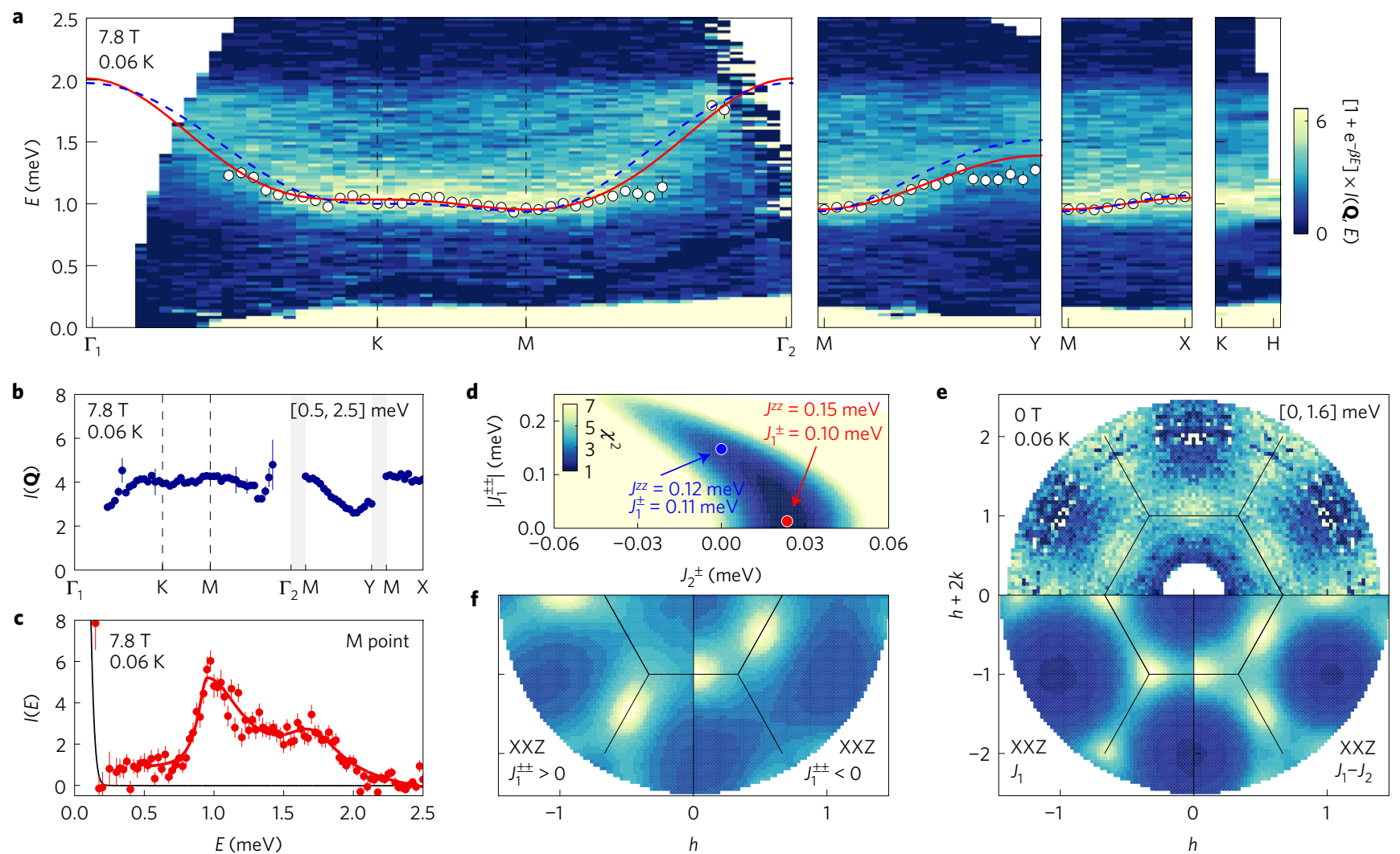

Figure 3 | Field-polarized neutron-scattering data and evidence for next-nearest-neighbour interactions in $\mathrm{YbMgGaO}_{4}$. $\mathbf{a}$, Energy dependence of magnetic excitations along high-symmetry directions, measured at $0.06 \mathrm{~K}$ in an applied field of $7.8 \mathrm{~T}$. The white circles show the location of the maximum intensity at each wavevector (see text). The red and blue lines show a fit to the spin-wave dispersion relation, equation (2). The labelling of reciprocal-space positions is given in Fig. 2b. The rightmost panel, for which $\mathrm{H} \equiv\left(\frac{1}{3} \frac{1}{3} \frac{1}{2}\right)$, demonstrates the absence of dispersion along I (perpendicular to the triangular planes). $\mathbf{b}$, One-dimensional plots of the $0.06 \mathrm{~K} E$-integrated magnetic signal, showing that the intensity is roughly constant along the chosen path, with the vicinity of the $Y$ point as a notable exception. $\mathbf{c}$, Representative fit to the $E$-dependence of the data shown in $\mathbf{a}$, used to determine the position of the intensity maximum at a given wavevector (white circles in a). d, Dependence of the goodness-of-fit parameter $\chi^{2}$ on the values of the exchange interactions $\left|J_{1}^{ \pm \pm}\right|$and $J_{2}^{ \pm}$, and two sets of exchanges discussed in the main text. e, Experimental diffuse-scattering data at $0.06 \mathrm{~K}$ obtained by integrating over energy transfer (top panel); calculation from classical Monte Carlo simulations of our $\left|J_{1}^{ \pm \pm}\right|=0$ model with XXZ nearest-neighbour interactions only (bottom left panel); and calculation including next-nearest-neighbour interactions (bottom right panel). $\mathbf{f}$, Calculated diffuse scattering for models with nearest-neighbour interactions only and $\left|J_{1}^{ \pm \pm}\right| / J_{1}^{ \pm}=1.36$ with $J_{1}^{ \pm \pm}>0$ (right panel) and $J_{1}^{ \pm \pm}<0$ (left panel) at a temperature of $5 \mathrm{~K}$. The data in a-c were measured with $E_{\mathrm{i}}=3.32 \mathrm{meV}$, and the data in e with $E_{\mathrm{i}}=12 \mathrm{meV}$. Error bars correspond to 1 standard error.

energy $(\sim 0.25 \mathrm{meV})$ shifts to between $\Gamma \equiv(000)$ and $\mathrm{K} \equiv\left(\frac{1}{3} \frac{1}{3} 0\right)$ points (Fig. 2c).

What is the probable origin of the QSL behaviour of $\mathrm{YbMgGaO}_{4}$ ? To answer this question, we measured its spectrum with a large magnetic field applied along the $c$ axis. The field polarizes the spins, yielding excitations that can be modelled by conventional linear spin-wave theory to determine the values of the exchange interactions ${ }^{21}$. The starting point for our analysis is the effective spin-1/2 Hamiltonian ${ }^{4,21,28}$,

$$
\begin{aligned}
\mathcal{H}= & \sum_{\langle i, j\rangle}\left[J_{1}^{z z} S_{i}^{z} S_{j}^{z}+J_{1}^{ \pm}\left(S_{i}^{+} S_{j}^{-}+S_{i}^{-} S_{j}^{+}\right)+J_{1}^{ \pm \pm}\left(\gamma_{i j} S_{i}^{+} S_{j}^{+}+\gamma_{i j}^{*} S_{i}^{-} S_{j}^{-}\right)\right] \\
& +\sum_{\langle\langle i, j\rangle\rangle}\left[J_{2}^{z z} S_{i}^{z} S_{j}^{z}+J_{2}^{ \pm}\left(S_{i}^{+} S_{j}^{-}+S_{i}^{-} S_{j}^{+}\right)\right] \\
& -\mu_{0} \mu_{\mathrm{B}} \sum_{i}\left[g_{\perp}\left(H^{x} S_{i}^{x}+H^{y} S_{i}^{y}\right)+g_{\|} H^{z} S_{i}^{z}\right]
\end{aligned}
$$

where spin operators $S^{ \pm}=S^{x} \pm \mathrm{i} S^{y}, g_{\|}$and $g_{\perp}$ are the components of the $g$-tensor parallel and perpendicular to the applied field, and the complex numbers $\gamma_{i j}$ are defined in ref. 4 . Brackets \langle\rangle and $\langle\langle\rangle\rangle$ denote nearest- and next-nearest-neighbour pairs, respectively. The exchange interactions $J^{z z}$ and $J^{ \pm}$define an XXZ model ${ }^{29}$, where we include nearest- and next-nearest-neighbour interactions (denoted by subscripts 1 and 2, respectively). We also include a symmetryallowed bond-dependent (also known as pseudo-dipolar) interaction, $J_{1}^{ \pm \pm}$; however, we neglect the other bond-dependent interaction $\left(J_{1}^{z \pm}\right.$ in ref. 4), because our current experimental data are insensitive to this term and ESR measurements indicate that it takes a very small value, $0.003(9) \mathrm{meV}$ (ref. 4). For a large applied field along $c$, it follows from equation (1) that the spin-wave dispersion is given by ${ }^{13,14}$

$$
\begin{aligned}
{[\varepsilon(\mathbf{Q})]^{2}=} & \left\{g_{\|} \mu_{\mathrm{B}} \mu_{0} H^{z}-3\left(J_{1}^{z z}+J_{2}^{z z}\right)\right. \\
& \left.+\sum_{i=1}^{6}\left[J_{1}^{ \pm} \cos \left(\mathbf{Q} \cdot \mathbf{r}_{1, i}\right)+J_{2}^{ \pm} \cos \left(\mathbf{Q} \cdot \mathbf{r}_{2, i}\right)\right]\right\}^{2} \\
& -\left|J_{1}^{ \pm \pm} \sum_{i=1}^{6} \gamma_{1, i}^{*} \cos \left(\mathbf{Q} \cdot \mathbf{r}_{1, i}\right)\right|^{2}
\end{aligned}
$$

where $\mathbf{r}_{1, i}$ and $\mathbf{r}_{2, i}$ label the nearest and next-nearest-neighbour vectors, respectively. 
Our experimental data measured in a $7.8 \mathrm{~T}$ field close to the $\approx 8 \mathrm{~T}$ saturation are shown in Fig. 3a. As anticipated from equation (2) and from the field dependence of the specific heat (Fig. 1b), the applied field induces a spin gap of $1.0 \mathrm{meV}$, and a single dispersive signal with a bandwidth of $1.1 \mathrm{meV}$ dominates the spectrum. A continuum of excitations is also visible and carries a significant spectral weight. This is a surprising observation that requires further investigation; we postulate that the continuum originates from exchange disorder induced by a distribution of local environments across $\mathrm{Yb}^{3+}$ ions. Nevertheless, two observations confirm that the system is essentially polarized: the integrated intensity of the inelastic scattering at $7.8 \mathrm{~T}$ is relatively constant throughout the Brillouin zone (Fig. 3b), and the overall intensity is significantly reduced compared with $0 \mathrm{~T}$, as expected for a field-induced ferromagnetic state ${ }^{30}$.

To model the dispersion, we first fit the $E$-dependence at each $\mathbf{Q}$ with a double (that is, asymmetric) Lorentzian to determine the peak position; a representative fit is shown in Fig. 3c. We then fit equation (2) to the extracted dispersion curve by varying the exchange parameters $J_{1}^{z z}+J_{2}^{z z}, J_{1}^{ \pm},\left|J_{1}^{ \pm \pm}\right|$, and $J_{2}^{ \pm}$. The choice of a purely two-dimensional model is justified by the absence of visible dispersion along $l$ (Fig. 3a). Throughout, we fix $g_{\|}=3.721$ from magnetization data ${ }^{4}$. Initially, we also fix $\left|J_{1}^{ \pm \pm}\right|=0.013 \mathrm{meV}$ from ESR measurements ${ }^{4}$. We obtain an excellent fit with $J_{1}^{z z}+$ $J_{2}^{z z}=0.154(3), J_{1}^{ \pm}=0.109(4)$ and $J_{2}^{ \pm}=0.024(1) \mathrm{meV}$. The ratio $J^{z z} / J^{ \pm}=1.16(4)$ is comparable to the result from magnetization measurements $\left(J^{z z} / J^{ \pm}=1.09(13)\right.$; ref. 4), locating the interactions of $\mathrm{YbMgGaO}_{4}$ between isotropic and planar limits $\left(J^{z z} / J^{ \pm}\right.$equal to 2 and 0 , respectively). In the limit of planar nearest-neighbour interactions, a gapless Dirac QSL may be stabilized ${ }^{31}$; however, this phase does not persist for $J_{1}^{z z} / J_{1}^{ \pm}>0$ (ref. 29). The parameters $J_{1}^{ \pm \pm}$and $J_{2}^{ \pm}$are therefore of particular interest, because both may in principle stabilize a QSL ${ }^{12-14}$. Figure $3 \mathrm{~d}$ shows the dependence of the goodness-of-fit on these parameters. Our dispersion curve is relatively insensitive to $\left|J_{1}^{ \pm \pm}\right|$: the best fit is for $\left|J_{1}^{ \pm \pm}\right|=0$, but the previously reported value of $0.013 \mathrm{meV}$ (ref. 4) yields visually indistinguishable results. Crucially, however, our data are highly sensitive to $J_{2}^{ \pm}$. Matching the shallow dispersion minimum at the $\mathrm{M}$ point yields a best-fit ratio $J_{2}^{ \pm} / J_{1}^{ \pm} \approx 0.22$ (Fig. 3a, solid red line). It is possible to reproduce this minimum without $J_{2}^{ \pm}$(ref. 13,14), but for fixed planar anisotropy $J^{z z} / J^{ \pm}=1.1$ this yields a large and dominant $\left|J_{1}^{ \pm \pm}\right|=0.15$ (1) meV (Fig. 3a, blue dashed line), contradicting the ESR result $\left(\left|J_{1}^{ \pm \pm}\right|=0.013(1) \mathrm{meV}\right.$ ) (ref. 4). Remarkably, the ratio $J_{2}^{ \pm} / J_{1}^{ \pm}$lies close to the QSL regime of the spin-1/2 $J_{1}-J_{2}$ Heisenberg triangular-lattice antiferromagnet, which is predicted to occur for $0.06 \lesssim J_{2} / J_{1} \lesssim 0.19$ (refs 9-12). Taken together with the published ESR results ${ }^{4}$, our $7.8 \mathrm{~T}$ results thus point towards moderate planar anisotropy with small antiferromagnetic next-nearest-neighbour $J_{2}^{ \pm}$ as a minimal model for $\mathrm{YbMgGaO}_{4}$.

Instantaneous spin-pair correlations in zero applied field provide an independent check on our results. We obtain the experimental diffuse intensity $I(\mathbf{Q})=\int_{0}^{E^{\prime}}\left(1+\mathrm{e}^{-\beta E}\right) I(\mathbf{Q}, E) \mathrm{d} E$, where $E^{\prime}=1.6 \mathrm{meV}$, and compare these data with classical Monte Carlo simulations in Fig. 3e,f. Our calculations, driven by equation (1), use our fitted values of the XXZ exchange interactions and assume $J_{1}^{z z} / J_{1}^{ \pm}=J_{2}^{z z} / J_{2}^{ \pm}$, where relevant. At a simulation temperature of $1.3 \mathrm{~K}$, the calculated diffuse intensity for $J_{2}^{ \pm}=J_{1}^{ \pm \pm}=0$ has its maximum at the $\mathrm{K}$ point, contrary to experiment. However, including $J_{2}^{ \pm}=0.22 J_{1}^{ \pm}$reproduces the M-point maximum observed experimentally (Fig. 3e) - a result also observed in quantum calculations ${ }^{12}$. A dominant $J_{1}^{ \pm \pm}$also produces M-point scattering ${ }^{4}$ but introduces an additional intensity modulation that depends on the sign of $J_{1}^{ \pm \pm}$, and is not observed in our experimental data (Fig. $3 \mathrm{f}$ and Supplementary Fig. 6). Crucially, after cooling our Monte Carlo simulations below $1.3 \mathrm{~K}$, the calculated diffuse scattering shows much sharper features than the experimental data (Supplementary Fig. 7). Quantum fluctuations are thus a possible contender to explain the suppressed spin correlation length of $2.8 \AA$ we observe at $0.06 \mathrm{~K}$.

Our observations in $\mathrm{YbMgGaO}_{4}$ set it apart from other inorganic triangular-lattice magnets with quantum spins, in which magnetic order or spin freezing typically occurs at a temperature $\sim \theta_{\mathrm{W}} / 10$ (see, for example, refs 25,32). In contrast, $\mathrm{YbMgGaO}_{4}$ shows a continuous excitation spectrum without magnetic order to temperatures below $\sim \theta_{\mathrm{W}} / 60$. Our results identify magnetic scattering at the $\mathrm{M}$ point of the hexagonal Brillouin zone and provide strong constraints on spin-space anisotropies and next-nearest-neighbour interactions to guide further theoretical investigations. Our work also calls for further studies to elucidate the effect of interstitial $\mathrm{Mg}^{2+} / \mathrm{Ga}^{3+}$ disorder on exchange interactions. Compared with molecule-based triangular QSL candidates ${ }^{33-35}$, the strength of the magnetic signal and the availability of large single-crystal samples make $\mathrm{YbMgGaO}_{4}$ an exceptional candidate for neutron-scattering experiments, suggesting that mapping the response of a twodimensional QSL candidate to temperature, applied magnetic field, and chemical perturbations is now a practical prospect.

\section{Methods}

Methods, including statements of data availability and any associated accession codes and references, are available in the online version of this paper.

Received 25 June 2016; accepted 1 November 2016; published online 5 December 2016

\section{References}

1. Balents, L. Spin liquids in frustrated magnets. Nature 464, 199-208 (2010)

2. Lee, P. A. An end to the drought of quantum spin liquids. Science 321, 1306-1307 (2008).

3. Li, Y. et al. Gapless quantum spin liquid ground state in the two-dimensional spin-1/2 triangular antiferromagnet $\mathrm{YbMgGaO}_{4}$. Sci. Rep. 5, 16419 (2015).

4. Li, Y. et al. Rare-earth triangular lattice spin liquid: a single-crystal study of $\mathrm{YbMgGaO}_{4}$. Phys. Rev. Lett. 115, 167203 (2015).

5. Li, Y. et al. Muon spin relaxation evidence for the $\mathrm{U}(1)$ quantum spin-liquid ground state in the triangular antiferromagnet $\mathrm{YbMgGaO}_{4}$. Phys. Rev. Lett. 117, 097201 (2016).

6. Shen, Y. et al. Spinon Fermi surface in a triangular lattice quantum spin liquid $\mathrm{YbMgGaO}_{4}$. Preprint at http://arXiv.org/abs/1607.02615 (2016).

7. Han, T.-H. et al. Fractionalized excitations in the spin-liquid state of a kagome-lattice antiferromagnet. Nature 492, 406-410 (2012).

8. Capriotti, L., Trumper, A. E. \& Sorella, S. Long-range Néel order in the triangular Heisenberg model. Phys. Rev. Lett. 82, 3899-3902 (1999).

9. Manuel, L. O. \& Ceccatto, H. A. Magnetic and quantum disordered phases in triangular-lattice Heisenberg antiferromagnets. Phys. Rev. B 60, 9489-9493 (1999).

10. Li, P. H. Y., Bishop, R. F. \& Campbell, C. E. Quasiclassical magnetic order and its loss in a spin-1/2 Heisenberg antiferromagnet on a triangular lattice with competing bonds. Phys. Rev. B 91, 014426 (2015).

11. Zhu, Z. \& White, S. R. Spin liquid phase of the spin- $1 / 2 J_{1}-J_{2}$ Heisenberg model on the triangular lattice. Phys. Rev. B 92, 041105 (2015).

12. Iqbal, Y., Hu, W.-J., Thomale, R., Poilblanc, D. \& Becca, F. Spin liquid nature in the Heisenberg $J_{1}-J_{2}$ triangular antiferromagnet. Phys. Rev. B 93, $144411(2016)$

13. Li, Y.-D., Wang, X. \& Chen, G. Anisotropic spin model of strong spin-orbit-coupled triangular antiferromagnets. Phys. Rev. B 94, 035107 (2016).

14. Li, Y.-D., Shen, Y., Li, Y., Zhao, J. \& Chen, G. The effect of spin-orbit coupling on the effective-spin correlation in $\mathrm{YbMgGaO}_{4}$. Preprint at http://arXiv.org/abs/1608.06445 (2016).

15. Savary, L. \& Balents, L. Disorder-induced entanglement in spin ice pyrochlores. Preprint at http://arXiv.org/abs/1604.04630 (2016).

16. Tennant, D. A., Perring, T. G., Cowley, R. A. \& Nagler, S. E. Unbound spinons in the spin-1/2 antiferromagnetic chain $\mathrm{KCuF}_{3}$. Phys. Rev. Lett. 70, 4003-4006 (1993)

17. Coldea, R., Tennant, D. A., Tsvelik, A. M. \& Tylczynski, Z. Experimental realization of a 2D fractional quantum spin liquid. Phys. Rev. Lett. 86, 1335-1338 (2001).

18. Banerjee, A. et al. Proximate Kitaev quantum spin liquid behaviour in a honeycomb magnet. Nat. Mater. 15, 733-740 (2016). 
19. Anderson, P. W. Resonating valence bonds: a new kind of insulator? Mater. Res. Bull. 8, 153-160 (1973).

20. de Vries, M. A. et al. Scale-free antiferromagnetic fluctuations in the spin-1/2 kagome antiferromagnet herbertsmithite. Phys. Rev. Lett. 103, 237201 (2009).

21. Ross, K. A., Savary, L., Gaulin, B. D. \& Balents, L. Quantum excitations in quantum spin ice. Phys. Rev. X 1, 021002 (2011)

22. Misguich, G., Lhuillier, C., Bernu, B. \& Waldtmann, C. Spin-liquid phase of the multiple-spin exchange Hamiltonian on the triangular lattice. Phys. Rev. B 60, 1064-1074 (1999).

23. Yaouanc, A., Dalmas de Réotier, P., Marin, C. \& Glazkov, V. Single-crystal versus polycrystalline samples of magnetically frustrated $\mathrm{Yb}_{2} \mathrm{Ti}_{2} \mathrm{O}_{7}$ : specific heat results. Phys. Rev. B 84, 172408 (2011).

24. Marshall, W. \& Lowde, R. D. Magnetic correlations and neutron scattering. Rep. Prog. Phys. 31, 705-775 (1968).

25. Ma, J. et al. Static and dynamical properties of the spin-1/2 equilateral triangular-lattice antiferromagnet $\mathrm{Ba}_{3} \mathrm{CoSb}_{2} \mathrm{O}_{9}$. Phys. Rev. Lett. 116, 087201 (2016).

26. Yan, S., Huse, D. A. \& White, S. R. Spin-liquid ground state of the spin-1/2 kagome Heisenberg antiferromagnet. Science 332, 1173 (2011).

27. Ross, K. A., Krizan, J. W., Rodriguez-Rivera, J. A., Cava, R. J. \& Broholm, C. L. Static and dynamic XY-like short-range order in a frustrated magnet with exchange disorder. Phys. Rev. B 93, 014433 (2016).

28. Onoda, S. Effective quantum pseudospin-1/2 model for $\mathrm{Yb}$ pyrochlore oxides. J. Phys. Conf. Ser. 320, 012065 (2011).

29. Yamamoto, D., Marmorini, G. \& Danshita, I. Quantum phase diagram of the triangular-lattice XXZ model in a magnetic field. Phys. Rev. Lett. 112 127203 (2014).

30. Mourigal, M. et al. Fractional spinon excitations in the quantum Heisenberg antiferromagnetic chain. Nat. Phys. 9, 435-441 (2013).

31. Alicea, J., Motrunich, O. I. \& Fisher, M. P. A. Algebraic vortex liquid in spin-1/2 triangular antiferromagnets: scenario for $\mathrm{Cs}_{2} \mathrm{CuCl}_{4}$. Phys. Rev. Lett. 95, 247203 (2005).

32. Nakatsuji, S. et al. Spin disorder on a triangular lattice. Science 309, 1697-1700 (2005).
33. Shimizu, Y., Miyagawa, K., Kanoda, K., Maesato, M. \& Saito, G. Spin liquid state in an organic Mott insulator with a triangular lattice. Phys. Rev. Lett. 91, 107001 (2003)

34. Pratt, F. L. et al. Magnetic and non-magnetic phases of a quantum spin liquid. Nature 471, 612-616 (2011)

35. Sheckelton, J. P., Neilson, J. R., Soltan, D. G. \& McQueen, T. M. Possible valence-bond condensation in the frustrated cluster magnet $\mathrm{LiZn}_{2} \mathrm{Mo}_{3} \mathrm{O}_{8}$. Nat Mater. 11, 493-496 (2012).

\section{Acknowledgements}

We are very grateful to L. Ge for his help with heat-capacity measurements and J. Carruth, S. Elorfi, M. Everett and C. Fletcher for sample environment and instrument support during our neutron-scattering experiments. It is our pleasure to thank S. Chernyshev, R. Coldea, K. Ross, M. Waterbury, Y. Wan and M. Zhitomirsky for insightful discussions The work and equipment at the Georgia Institute of Technology (J.A.M.P., M.D. and M.M.) was supported by the College of Sciences and the Executive Vice-President for Research. The work at the University of Tennessee (Z.D. and H.Z.) was supported by the National Science Foundation through award DMR-1350002. The research at Oak Ridge National Laboratory's Spallation Neutron Source was sponsored by the US Department of Energy, Office of Basic Energy Sciences, Scientific User Facilities Division.

\section{Author contributions}

J.A.M.P., M.D., Z.D., G.E., Y.L., M.B.S. and M.M. performed neutron-scattering experiments. J.A.M.P., M.D. and M.M. analysed the data. Z.D. and H.Z. made the sample. Z.D. and M.M. characterized the sample. M.D. and M.M. aligned the sample. M.M. made the figures and J.A.M.P. wrote the paper with input from all authors. H.Z. and M.M. designed and supervised the project.

\section{Additional information}

Supplementary information is available in the online version of the paper. Reprints and permissions information is available online at www.nature.com/reprints. Correspondence and requests for materials should be addressed to M.M.

\section{Competing financial interests}

The authors declare no competing financial interests. 


\section{Methods}

Sample preparation. A polycrystalline sample of $\mathrm{YbMgGaO}_{4}$ was synthesized by a solid-state method. Stoichiometric ratios of $\mathrm{Yb}_{2} \mathrm{O}_{3}, \mathrm{MgO}$ and $\mathrm{Ga}_{2} \mathrm{O}_{3}$ fine powder were carefully ground and reacted at a temperature of $1,450^{\circ} \mathrm{C}$ for 3 days with several intermediate grindings. The single-crystal sample of $\mathrm{YbMgGaO}_{4}$

(Supplementary Fig. 2) was grown using the optical floating-zone method under a $5 \mathrm{~atm}$ oxygen atmosphere ${ }^{4}$. The best single crystal was obtained with a pulling speed of $1.5 \mathrm{~mm} \mathrm{~h}^{-1}$, and showed cliffed [001] surfaces after several hours of growth.

$\mathrm{X}$-ray diffraction measurements and refinements. Room-temperature powder $\mathrm{X}$-ray diffraction were carried out on powder and crushed single-crystal samples using a Panalytical X'pert Pro Alpha-1 diffractometer with monochromatic $\mathrm{Cu}-\mathrm{K} \alpha$ radiation $(\lambda=1.540598 \AA)$. Preliminary measurements in flat-plate geometry on a loose powder using a Huber X-ray diffractometer showed preferred orientation, especially for the crushed-crystal sample. To minimize the extent of preferred orientation, we loaded our samples into a glass capillary that was rotated at 30 r.p.m. Due to the large absorption cross-section of Yb, these measurements were limited to small sample sizes. Measurements were taken between $5 \leq 2 \theta \leq 140^{\circ}$ with $\Delta 2 \theta=0.016^{\circ}$. Rietveld refinement was carried out using the FULLPROF program $^{36}$. Peak shapes were modelled by pseudo-Voigt functions, and the remaining preferred orientation was treated within the March model ${ }^{37}$. Fits to data are shown in Supplementary Fig. 4, and refined values of structural parameters are given in Supplementary Table 1.

Thermomagnetic measurements. Heat-capacity measurements were carried out on a Quantum Design physical property measurement system (PPMS) instrument using dilution fridge $(0.06 \leq T \leq 4 \mathrm{~K})$ and standard $(1.6 \leq T \leq 100 \mathrm{~K})$ probes in a range of measuring magnetic fields, $0 \leq \mu_{0} H \leq 14 \mathrm{~T}$. Single-crystal measurements were made on a flat thin piece polished to $\approx 1 \mathrm{mg}$ and oriented with the $c$ axis parallel to the applied magnetic field. To ensure sample thermalization at low temperatures, powder measurements were made on pellets of $\mathrm{YbMgGaO}_{4}$ mixed with an approximately equal mass of silver powder, the contribution of which was measured separately and subtracted to obtain the specific heat $C_{\mathrm{p}}$. The magnetic specific heat $C_{\mathrm{m}}$ was obtained by subtracting a modelled lattice contribution $C_{\mathrm{L}}$ with two Debye temperatures, $480 \mathrm{~K}$ and $142 \mathrm{~K}$. The change in magnetic entropy $\Delta S(T)$ was subsequently obtained by integrating $C_{\mathrm{m}} / T$ from $0.06 \mathrm{~K}$ to $T$. Isothermal magnetization measurements $M(H)$ were performed on the above single-crystal piece using a PPMS vibrating sample magnetometer in a range of magnetic fields $0 \leq \mu_{0} H \leq 14 \mathrm{~T}$ and temperatures $1.7 \leq T \leq 10 \mathrm{~K}$.

Low-energy inelastic neutron-scattering measurements. Inelastic neutron-scattering ${ }^{24}$ experiments were performed on the cold neutron chopper spectrometer (CNCS) at the Spallation Neutron Source (SNS), Oak Ridge National Laboratory ${ }^{38}$. The sample was a $2.2 \mathrm{~g}$ rod-shaped crystal cut into two shorter pieces to fit in the bore of a cryomagnet. The two pieces (total dimensions $16 \times 16 \times 4 \mathrm{~mm}^{3}$ ) were co-aligned to within $1.5^{\circ}$ using a Multiwire X-ray Laue backscattering machine, and mounted in the ( $h k 0)$ scattering plane on a oxygen-free copper holder (Supplementary Fig. 2). The mount was attached to the bottom of a dilution refrigerator reaching a base temperature of $0.06 \mathrm{~K}$ at the mixing chamber, indicating a sample temperature $\lesssim 0.1 \mathrm{~K}$. The sample stick was inserted in an $8 \mathrm{~T}$ superconducting cryomagnet, and measurements were performed in zero field and in a field of 7.8 T applied along the $c$ axis. Two incident neutron energies were used, $E_{\mathrm{i}}=3.32$ and $12.0 \mathrm{meV}$, yielding elastic energy resolutions (full-width at half-maximum) of 0.11 and $0.75 \mathrm{meV}$, respectively. The sample was rotated in steps of $1^{\circ}$, with a range of $270^{\circ}$ for $E_{\mathrm{i}}=12 \mathrm{meV}$ and $180^{\circ}$ for $E_{\mathrm{i}}=3.32 \mathrm{meV}$. For $E_{\mathrm{i}}=3.32 \mathrm{meV}$, the background scattering from the sample environment was measured and subtracted from the data. For $E_{\mathrm{i}}=12 \mathrm{meV}$, the background and non-magnetic scattering at low energy $(E \leq 0.9 \mathrm{meV})$ was subtracted using the 7.8 T measurement, taking advantage of the spin gap induced by applied field.

Broadband neutron-scattering measurements. Broadband neutron-scattering experiments were performed on a sintered powder sample and on one of the above single crystals using the SEQUOIA spectrometer at ORNL's SNS ${ }^{39}$. The powder sample was mounted in a cylindrical aluminium can sealed under one atmosphere of ${ }^{4} \mathrm{He}$ at room temperature. The single crystal was mounted with its $a b$ plane horizontal and rotated continuously, yielding an effective two-dimensional powder averaging. Both samples were cooled using a closed-cycle refrigerator reaching a base temperature of $T=4 \mathrm{~K}$. The spectrometer was operated with three distinct configurations to cover a large range of momentum and energy transfers: $E_{\mathrm{i}}=300 \mathrm{meV}$ with a Fermi chopper frequency of $f=420 \mathrm{~Hz}, E_{\mathrm{i}}=160 \mathrm{meV}$ with $f=600 \mathrm{~Hz}$, and $E_{\mathrm{i}}=22 \mathrm{meV}$ with $f=300 \mathrm{~Hz}$. Background and sample holder contributions were subtracted from the data using empty can measurements. The contribution from the lattice was subtracted by extracting the phonon density of states at $|\mathbf{Q}|=6.0 \AA^{-1}(160 \mathrm{meV})$ and $|\mathbf{Q}|=9.0 \AA^{-1}(300 \mathrm{meV})$ and extrapolating to other momenta using the known $|\mathbf{Q}|^{2}$ intensity dependence for single-phonon scattering ${ }^{40}$. The position and width of the observed excitations were obtained through fits to Gaussian profiles. The energy resolution of the spectrometer was estimated using simplified geometrical considerations yielding a correct elastic energy resolution but known to under-estimate the energy resolution at finite energy transfer. Thirteen electrons reside in the $4 f$ shell of the Kramers ion $\mathrm{Yb}^{3+}$ Following ref. 41, the crystal electric field can maximally split the local electronic manifold into four Kramers doublets yielding three excited doublets above the doublet ground state.

Elastic neutron-scattering measurements. Elastic neutron-scattering experiments were performed on the CORELLI spectrometer at ORNL's SNS. One of the two above crystal pieces was attached to a copper pin at the bottom of a ${ }^{3} \mathrm{He}$ cryostat reaching a base temperature of $0.3 \mathrm{~K}$. The sample was aligned in the ( $h 0 l$ ) scattering plane to assess crystal quality and stacking of the triangular-lattice planes along $c$. Neutron-absorbing $\mathrm{Cd}$ was used to shield the sample holder and an empty cryostat measurement was used to remove the background contribution. The sample was rotated in steps of $6^{\circ}$ over a $360^{\circ}$ range, and measurements were taken at temperatures of $0.3,4.0$ and $40 \mathrm{~K}$. Elastic neutron-scattering data measured at $0.3 \mathrm{~K}$ are shown in Supplementary Fig. 3 .

Data analysis. Initial data reduction was performed in MANTID ${ }^{42}$ for CNCS, SEQUOIA and CORELLI data sets. For the CNCS data, subsequent analysis was performed in HORACE ${ }^{43}$ on a dedicated node within Georgia Tech's Partnership for Advanced Computing infrastructure. To increase counting statistics, inelastic-scattering data were symmetrized into an irreducible $60^{\circ}$ wedge of the hexagonal reciprocal lattice. The CORELLI data were normalized to absolute units in MANTID ${ }^{44}$.

Monte Carlo simulations. To perform classical Monte Carlo simulations, we rewrite the spin Hamiltonian, equation (1), for zero field in terms of spin components $S^{x}, S^{y}$ and $S^{z}$ :

$$
\begin{aligned}
H= & \sum_{\langle i, j\rangle}\left\{J_{1}^{z z} S_{i}^{z} S_{j}^{z}+2 J_{1}^{ \pm}\left(S_{i}^{x} S_{j}^{x}+S_{i}^{y} S_{j}^{y}\right)+2 J_{1}^{ \pm \pm}\left[\left(S_{i}^{x} S_{j}^{x}-S_{i}^{y} S_{j}^{y}\right) \cos \left(\phi_{i j}\right)\right.\right. \\
& \left.\left.-\left(S_{i}^{x} S_{j}^{y}+S_{i}^{y} S_{j}^{x}\right) \sin \left(\phi_{i j}\right)\right]\right\}+\sum_{\langle\langle i, j\rangle}\left[J_{2}^{z z} S_{i}^{z} S_{j}^{z}+2 J_{2}^{ \pm}\left(S_{i}^{x} S_{j}^{x}+S_{i}^{y} S_{j}^{y}\right)\right]
\end{aligned}
$$

where the phase factors $\phi_{i j}=\phi_{j i}=\left\{0,+\frac{2 \pi}{3},-\frac{2 \pi}{3}\right\}$ for nearest-neighbour bonds along the directions $\mathbf{a}, \mathbf{b}$ and $\mathbf{a}+\mathbf{b}$, respectively (where $\mathbf{a}$ and $\mathbf{b}$ are shown in Fig. 1a). In Fig. 3e, we keep $J_{1}^{z z}=0.126$ and $J_{1}^{ \pm}=0.109 \mathrm{meV}$ with either $J_{2}^{z z}=0$ and $J_{2}^{ \pm}=0$ (bottom left) or $J_{2}^{z z}=0.027$ and $J_{2}^{ \pm}=0.024 \mathrm{meV}$ (bottom right). In Fig. 3f, we take $J_{1}^{ \pm \pm}= \pm 0.15 \mathrm{meV}$ with $J_{1}^{z z}=0.12$ and $J_{1}^{ \pm}=0.11 \mathrm{meV}$. The effect of small non-zero $J_{1}^{ \pm \pm}= \pm 0.013 \mathrm{meV}$ is discussed in the Supplementary Information. We take the length of spin vectors as $\sqrt{S(S+1)}=\sqrt{3} / 2$. Simulations were performed on a three-dimensional spin configuration consisting of nine triangular layers, each containing 504 spins. A proposed spin move consists of rotating a spin by a small amount (chosen so that $50-70 \%$ of proposed moves were accepted). The simulations were initialized at a high temperature and cooled in gradual increments. At each temperature, the simulation was run for at least $10 t_{0}$ proposed moves, where $t_{0}$ is the number of proposed moves required to decorrelate the system. The single-crystal diffuse-scattering intensity was calculated as $^{40}$

$$
I(\mathbf{Q}) \propto[f(\mathrm{Q})]^{2}\left|\sum_{i} \mathbf{S}_{i}^{\perp} \exp \left(\mathrm{iQ} \cdot \mathbf{r}_{i}\right)\right|^{2}
$$

where $f(Q)$ is the $\mathrm{Yb}^{3+}$ magnetic form factor ${ }^{45}$, and $\mathbf{S}_{i}^{\perp}=\mathbf{S}_{i}-\mathbf{Q}\left(\mathbf{S}_{i} \cdot \mathbf{Q}\right) / Q^{2}$ is the component of spin $\mathbf{S}_{i}$ perpendicular to $\mathbf{Q}$. The calculated pattern was slightly smoothed to allow calculation on an arbitrary Q-grid. To increase statistical averaging, the calculated $I(\mathbf{Q})$ was averaged over 100 spin configurations and $\overline{3} m$ diffraction symmetry was applied.

Data availability. The data that support the plots within this paper and other findings of this study are available from the corresponding author on request.

\section{References}

36. Rodríguez-Carvajal, J. Recent advances in magnetic structure determination by neutron powder diffraction. Physica B 192, 55-69 (1993).

37. Dollase, W. A. Correction of intensities for preferred orientation in powder diffractometry: application of the March model. J. Appl. Crystallogr. 19, 267-272 (1986).

38. Ehlers, G., Podlesnyak, A. A., Niedziela, J. L., Iverson, E. B. \& Sokol, P. E. The new cold neutron chopper spectrometer at the Spallation Neutron Source: design and performance. Rev. Sci. Instrum. 82, 085108 (2011).

39. Granroth, G. E. et al. SEQUOIA: a newly operating chopper spectrometer at the SNS. J. Phys. Conf. Ser. 251, 12058 (2010).

40. Squires, G. L. Introduction to the Theory of Thermal Neutron Scattering 129-145 (Cambridge Univ. Press, 1978).

41. Gaudet, J. et al. Neutron spectroscopic study of crystalline electric field excitations in stoichiometric and lightly stuffed $\mathrm{Yb}_{2} \mathrm{Ti}_{2} \mathrm{O}_{7}$. Phys. Rev. B 92, 134420 (2015). 
42. Arnold, O. et al. Mantid-data analysis and visualization package for neutron scattering and $\mu$ SR experiments. Nucl. Instrum. Methods Phys. Res. A 764, 156-166 (2014).

43. Ewings, R. A. et al. HORACE: software for the analysis of data from single crystal spectroscopy experiments at time-of-flight neutron instruments. $\mathrm{Nucl}$. Instrum. Methods Phys. Res. A 884, 132-142 (2016).
44. Michels-Clark, T. M., Savici, A. T., Lynch, V. E., Wang, X. P. \& Hoffmann, C. M. Expanding Lorentz and spectrum corrections to large volumes of reciprocal space for single-crystal time-of-flight neutron diffraction. J. Appl. Crystallogr. 49, 497-506 (2016).

45. Brown, P. J. International Tables for Crystallography Vol. C, 454-460 (Kluwer-Academic, 2004) 